\title{
Advanced Glycation End Products Levels and Carotid Intima Media Thickness in Patients With Rheumatoid Arthritis
}

\author{
Ines KNANI
}

Research Unit UR17ES30, Higher Institute of Biotechnology, University of Monastir, Tunisia Hassan BOUZIDI

Laboratory of Biochemistry, University Hospital of Tahar Sfar, Mahdia, Tunisia

Albert LECUBE

Endocrinology and Nutrition Department, Hospital Universitari Arnau de Vilanova. Immunology and Metabolism Research Group (GRIM). Institut de Recerca Biomèdica (IRB) de Lleida. Universitat de Lleida. Lleida, Catalonia, Spain

Jawhar GHARBI

Research Unit UR17ES30, Higher Institute of Biotechnology, University of Monastir, Tunisia Mohsen KERKENI, PhD (Corresponding Author)

Higher Institute of Biotechnology, BP 74, Avenue Tahar Haddad - 5000 Monastir - Tunisie Tél: +216 73465 405; Fax: +216 73465 404; E-mail: mohsen.kerkeni@yahoo.fr

Received: May 7, 2019 Accepted: May 28, 2019

doi:10.5296/jbls.v10i2.14967 URL: https://doi.org/10.5296/jbls.v10i2.14967

\begin{abstract}
Introduction: No data regarding the relationship of carotid intima media thickness (IMT) and serum advanced glycation end products (AGEs) levels in patients with rheumatoid arthritis (RA). The aim of this study is to evaluate the association between IMT and serum pentosidine, CML and MGO levels in patients with longstanding RA.

Methods: In a cross-sectional study, 80 consecutive RA patients with longstanding disease were included and compared with 30 age and sex-matched healthy controls. IMT was measured using ultrasonography. AGEs such as pentosidine, $\mathrm{N}^{\varepsilon}$-carboxymethyllysine (CML) and methylglyoxal (MGO) as an intermediate of glycation, were determined by ELISA.
\end{abstract}

Results: Serum pentosidine, CML and MGO levels were increased in RA patients $v s$ control 
subjects $(P=0.001 ; P<0.001 ; P<0.001$ respectively). IMT was increased with disease activity of RA $(P=0.004)$ and was associated with serum pentosidine $(r=0.460, P<0.001)$, serum CML $(r=0.549, P<0.001)$ and serum MGO $(r=0.658, P<0.001)$. Furthermore, in a multiple stepwise regression analysis CML and MGO were independently associated with $\operatorname{IMT}(\beta=0.333, P=0.007 ; \beta=0.690, P<0.001$, respectively).

Conclusion: serum pentosidine, CML and MGO were increased in RA patients and were significantly related to IMT. Serum CML and MGO were independently associated with IMT.

Keywords: pentosidine, $\mathrm{N}^{\varepsilon}$-carboxymethyllysine, methylglyoxal, carotid intima media thickness, rheumatoid arthritis

\section{Introduction}

Rheumatoid arthritis (RA) is the most common inflammatory rheumatism whose origin is still mysterious and evolutionary. It affects 1 to $2 \%$ of adults in the world with a female predominance: three times more common among women than men. It occurs at any age with a higher prevalence between 40 and 50 years (Mateen, 2016). RA is a chronic systemic inflammatory disease characterized by premature death with increased cardiovascular disease (CVD) mortality (Kramer, 2011). The increased CVD morbidity and mortality in patients with RA cannot be entirely explained by traditional CVD risk factors and chronic inflammation seems to act as an independent CVD risk factor (Baghdadi, 2015; Agca, 2017). Chronic inflammation can lead to oxidative stress and develop the formation of reactive carbonyl compounds, which are transformed into advanced glycation end products (AGEs) by nonenzymatic glycation process (Nasiri, 2012).

Nonenzymatic glycation begins with interaction between the carbonyl group of a reducing sugar an amino group of proteins. Complex rearrangements results in more stable AGEs precursors or named intermediate AGEs, these compounds, progressively transformed by irreversible reactions in AGEs such as pentosidine and $\mathrm{N}^{\varepsilon}$-carboxymethyllysine (CML). Methylglyoxal (MGO), one of reactive carbonyl species, formed endogenously as a by-product of the glycolytic pathway, by degradation of triosphosphates or nonenzymatically by sugar fragmentation reactions, is the most potent precursor of AGEs formation, in particular, pentosidine formation (Nasiri, 2012). Previously, we showed that serum AGEs were implicated in many diseases such as diabetic microvascular and macrovascular disease, atherosclerosis and metabolic syndrome (Kerkeni, 2012, 2013, 2014, Haddad, 2016) and recently, we showed that CML and MGO were independently associated with disease activity in RA (Knani 2018a, 2018b).

Carotid intima media thickness (IMT) of the common carotid artery used to study early structural changes in the arterial wall and reflects early atherosclerosis and predicts CVD events in the general population, it is the most popular useful technique noninvasive anatomical structural measure of subclinical CVD (Gonzalez-Gay,2008). IMT is associated with systemic rheumatoid inflammation add to the cardiovascular phenomenon in RA (Ambrosino, 2015; Gasparyan, 2010; Im, 2015). To shed light, there are no studies about the relationship between serum AGEs levels and IMT in RA patients. For that, we investigate the 
association between serum pentosidine, CML, MGO and IMT in RA patients and how these could serve as biomarker for IMT.

\section{Materials and Methods}

\subsection{Patients and Controls}

This study was involved 80 patients without cardiovascular disease or nephropathy and with a clinical diagnosis of RA according to American College of Rheumatology criteria for rheumatoid arthritis (Aletaha 2010) who were recruited in the rheumatology department in University Hospital Fattouma Bourguiba Monastir (Tunisia) and 30 healthy controls with no past or family history of RA or associated autoimmune disease were enrolled in the study. All RA patients were subjected to history taking especially for presenting symptoms, joint affection, together with history of medications. Disease activity was used to assess disease activity Score for 28 joints (DAS28) according to EULAR's recommendations (Fransen 2005). Disability was assessed using the Health Assessment Questionnaire (HAQ), a score by questionnaire that examines the disabilities that RA patients encounter in daily living and activities (Kumar, 2002). The study was approved by the local medical ethics committee and informed consent was obtained from all study participants.

\subsection{Measurement of Carotid Intima Media Thickness (IMT)}

Carotid artery atherosclerosis was determined by ultrasonographic measurement of the IMT of the common carotid artery $1 \mathrm{~cm}$ proximal to the carotid artery bifurcation on both the left and right sides (Riley, 1992). IMT was defined as the distance from the leading edge of the media adventitia interface of the far wall. Ultrasonographic scanning was performed in simple blind by a single experienced ultrasonographer who was unaware of the clinical findings and who scanned the left and the right common carotid artery using a $10 \mathrm{MHz}$ linear array transducer (Esaote My Lab 50 ultrasonograph). The final IMT value represents an average of the IMT results from the left and the right sides.

\subsection{Biochemical Analysis}

In all subjects, blood was drawn in the morning after overnight fasting, and the serum were separated immediately and stored at $80^{\circ} \mathrm{C}$ until analysis. The enzymatic method, $\mathrm{CX} 9$ Auto-chemical analysis instrument (Beckman CX9, USA) was used to measure glucose, and lipid profile (Cholesterol, triglyceride, HDL and LDL). Glycated hemoglobin (HbA1c) was measured using G7 HPLC Analyser (Tososh Europe N.V). High-sensitive C-reactive protein (hs-CRP) was quantified according to the instructions of manufacturers using particles enhanced immunonephelomitric method (BNII, Dade Behring). Anti-cyclic citrullinated peptides (anti-CCP) were measured by a second generation ELISA (Euroimmun ${ }^{\circledR}$, Germany). $\operatorname{IgM}$ rheumatoid factor (IgM-RF) was measured by the Waaler-Rose and the latex agglutination test (Fumouze $\AA$, France). Pentosidine, CML, MGO were quantitatively determined in serum by ELISA kits provided by YH Biosearch Laboratory (Shanghai, China) according to the manufacturer's instructions. Pentosidine (or CML, or MGO) present in the sample was probed with an anti-pentosidine (or anti-CML, or anti-MGO) antibody, followed by a horseradish peroxidase-conjugated secondary antibody. Pentosidine or CML or MGO 


\section{Macrothink

content in the sample were determined by comparison with a standard curve prepared from pentosidine standards ranging $0-12 \mathrm{ng} / \mathrm{mL}$, or from CML standards ranging $0-160 \mathrm{ng} / \mathrm{mL}$, or from MGO standards ranging $0-320 \mathrm{ng} / \mathrm{mL}$. The absorbance of the final color product was read at $450 \mathrm{~nm}$ by a Bio Rad mutiplate reader (Model 680).

\subsection{Statistical Analysis}

All analyses were performed using SPSS version 17.0 (SPSS Inc., Chicago, IL, USA). Continuous variables were expressed as median (minimum to maximum) or (interquartile ranges), while percentages were used to express categorical variables. An unpaired Student's $t$-test was used for normally distributed variable, and the Mann-Whitney U-test was used for skewed variables. Spearman correlation analysis was performed to determine factors that correlated with IMT. To determine the independent parameters associated with IMT, the parameters that associated significantly in the univariate analysis were tested using multiple stepwise regression analysis. A $P<0.05$ was considered as statistically significant.

\section{Results}

\subsection{Clinical Characteristic of RA Patients}

The clinical characteristics of RA patients are shown in Table 1. DAS28 value was 5.56 (1.99 to 8.42 ) at the time of investigation. $41 \%$ of all RA patients had moderate disease activity, and 59\% had high disease activity. Mean erythrocyte sedimentation rate of 33 (5 to 100). Anti-CCP and IgM-RF were present in $62.5 \%$ and $68.7 \%$ of the cases, respectively. Mean IMT value was $0.75 \mathrm{~mm}(0.60$ to $1 \mathrm{~mm})$.

Table 1. Main clinical characteristics and metabolic data of patients with rheumatoid arthritis (RA) included in the study

\begin{tabular}{lc}
\hline & $\begin{array}{c}\text { RA patients } \\
\text { (n = 80) }\end{array}$ \\
\hline Female (\%) & 89 \\
Diabetes (\%) & 12 \\
Hypertension (\%) & 20 \\
Dyslipidemia (\%) & 64 \\
ESR (mm/H) & $33(5$ to 100$)$ \\
Disease duration (yrs) & $10(1$ to 37$)$ \\
Swollen joint count (SJC) & $0.5(0$ to 20$)$ \\
Tender joint count (TJC) & $14(0$ to 28$)$ \\
HAQ score & $2(1$ to 2.1$)$ \\
Anti-CCP $(\%)$ & 62.5 \\
IgM-RF (\%) & 68.7 \\
DAS28 & $5.56(1.99$ to 8.42$)$ \\
IMT (mm) & $0.75(0.60$ to 1$)$ \\
Moderate (\%) & 41 \\
Active (\%) & 59 \\
Medication & \\
Methotrexate (\%) & 85 \\
Leflunomide (\%) & 8.7 \\
Salazopyrin (\%) & 12.5 \\
\hline
\end{tabular}


Data are represented as median (minimum to maximum) or percentages. ESR: erythrocyte sedimentation rate; HAQ: health assessment questionnaire; CCP: cyclic citrullinated peptides; RF: rheumatoid factor; DAS28: disease activity Score for 28 joints.

\subsection{Biological Parameters and AGEs Levels in RA Patients and Control Subjects}

Biological parameters and serum AGEs levels of RA patients and control subjects are shown in Table 2. Significant differences in BMI and hs-CRP were seen between the groups. Triglyceride levels were significantly higher in RA patients compared to control subjects $(P<$ 0.001), whereas HDL levels were significantly lower compared to control subjects $(P<$ 0.001). Serum pentosidine, CML and MGO were increased in RA patients compared to control subjects $(P=0.001, P<0.001, P<0.001$ respectively $)$.

Table 2. Biological parameters and AGEs levels in RA patients and control subjects

\begin{tabular}{lccc}
\hline & $\begin{array}{c}\text { RA patients } \\
(\mathbf{n}=\mathbf{8 0})\end{array}$ & $\begin{array}{c}\text { Control subjects } \\
(\mathbf{n = 3 0})\end{array}$ & $\boldsymbol{P}$ \\
\hline BMI $\left(\mathbf{k g} / \mathbf{m}^{\mathbf{2}}\right)$ & $28.4(24.2-35)$ & $22.9(20.4-28.6)$ & $<0.001$ \\
CRP $(\mathbf{m g} / \mathbf{L})$ & $9.7(5-15.8)$ & $0(0-5)$ & $<0.001$ \\
Glucose (mmol/L) & $4.3(4.0-4.6)$ & $4.05(3.4-4.5)$ & $\mathrm{NS}$ \\
Triglyceride (mmol/L) & $1.4(1.0-2.1)$ & $0.9(0.7-1.4)$ & $<0.001$ \\
Cholesterol $(\mathbf{m m o l} / \mathbf{L})$ & $4.3(3.7-4.9)$ & $4.3(4.0-5.0)$ & $\mathrm{NS}$ \\
HDL $(\mathbf{m m o l} / \mathbf{L})$ & $0.9(0.8-1.3)$ & $1.3(1.1-1.6)$ & $<0.001$ \\
LDL $(\mathbf{m m o l} / \mathbf{L})$ & $2.5(2.1-3.0)$ & $2.4(2.3-3.8)$ & $\mathrm{NS}$ \\
Pentosidine (ng/mL) & $5.8(5.0-7.3)$ & $4.1(3.6-5.0)$ & 0.001 \\
CML $(\mathbf{n g} / \mathbf{m L})$ & $74.2(58.7-86.5)$ & $42.1(34.4-45.9)$ & $<0.001$ \\
MGO $(\mathbf{n g} / \mathbf{m L})$ & $117.1(83.2-177.1)$ & $72.6(53.3-85.7)$ & $<0.001$ \\
\hline
\end{tabular}

Data are represented as median (interquartile range). BMI: body mass index; CML: $\mathrm{N}^{\varepsilon}$ carboxymethyllysine; MGO: methylglyoxal. NS: not significant. 


\section{Macrothink}

\subsection{Serum AGEs and Carotid IMT Levels in RA Patients with Disease Activity}

AGEs and IMT levels in RA patients with disease activity are shown in Table 3. Pentosidine, CML and MGO and IMT levels were increased in patients with high DAS28 compared to the patients with moderate DAS28 $(P=0.019, P<0.001, P=0.012, P=0.004$ respectively $)$.

Table 3. Serum AGEs and IMT levels in RA patients with disease activity

\begin{tabular}{lccc}
\hline Parameters & $\begin{array}{c}\text { RA patients with moderate DAS28 } \\
(\mathbf{n}=\mathbf{3 3})\end{array}$ & $\begin{array}{c}\text { RA patients with } \\
\text { high DAS28 } \\
(\mathbf{n}=\mathbf{4 7})\end{array}$ & $\boldsymbol{P}$ \\
\hline Pentosidine $(\mathbf{n g} / \mathbf{m L})$ & $5.77(4.73-6.30)$ & $6.19(5.31-7.77)$ & 0.019 \\
CML $(\mathbf{n g} / \mathbf{m L})$ & $55.67(45.11-74.85)$ & $78.73(73.10-96.23)$ & $<0.001$ \\
MGO $(\mathbf{n g} / \mathbf{m L})$ & $77.28(66.17-112)$ & $108(78-121)$ & 0.012 \\
IMT $(\mathbf{m m})$ & $0.71(0.64-0.79)$ & $0.78(0.71-0.85)$ & 0.004 \\
\hline
\end{tabular}

Data are represented as median (interquartile range).

NS: not significant.

\subsection{Relationship Between Serum Pentosidine, CML, MGO and IMT in RA Patients}

Spearman correlation analysis showed that serum pentosidine, CML and MGO were positively correlated with IMT ( $r=0.460, P<0.001 ; r=0.549, P<0.001 ; r=0.658, P<0.001)$ as illustrated in figure 1, 2 and 3. Furthermore, a multiple stepwise regression analysis in order to determine the relationships between serum AGEs and IMT in a model adjusted for the following parameters: age, BMI, hypertension, glucose, triglyceride, cholesterol, HDL, LDL and hs-CRP. This analysis showed that serum CML and MGO were independently associated with IMT ( $\beta=0.333, P=0.007 ; \beta=0.690, P<0.001$, respectively).

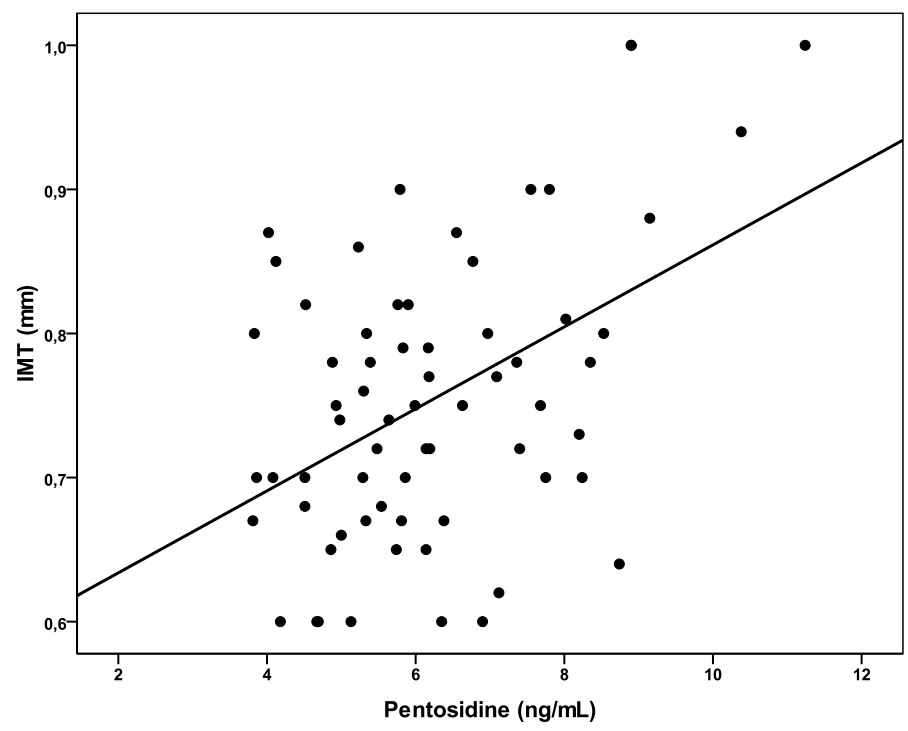

Figure 1. Scatter plot showing linear correlation between serum pentosidine and IMT in RA patients 


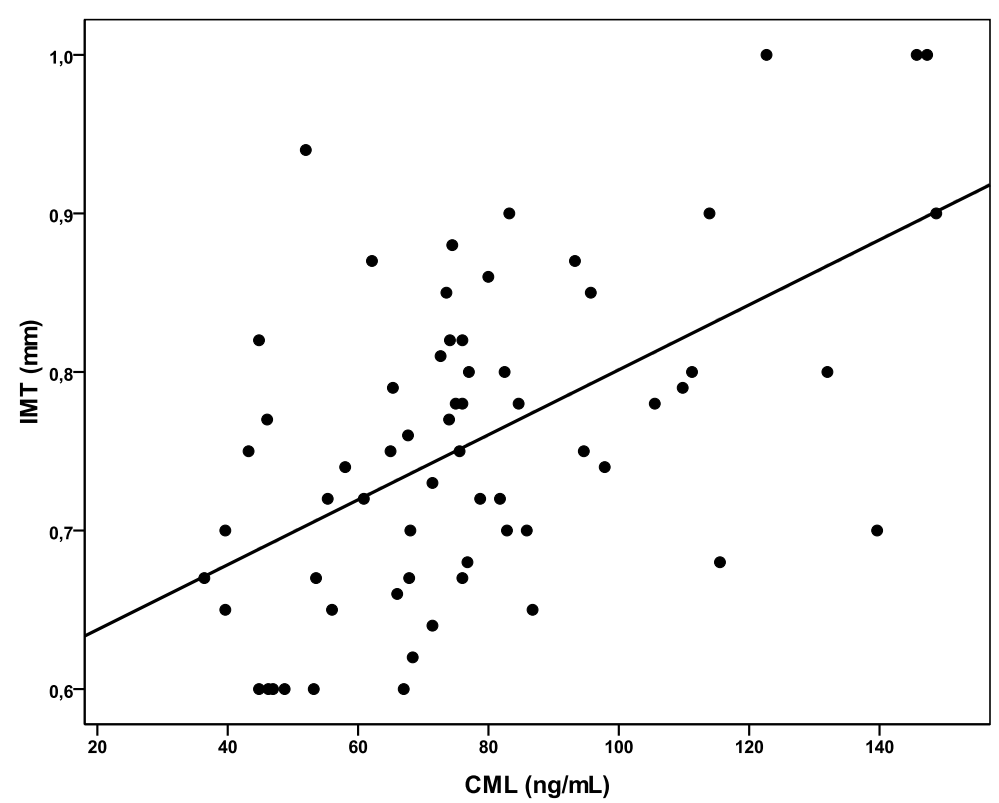

Figure 2. Scatter plot showing linear correlation between serum CML and IMT in RA patients

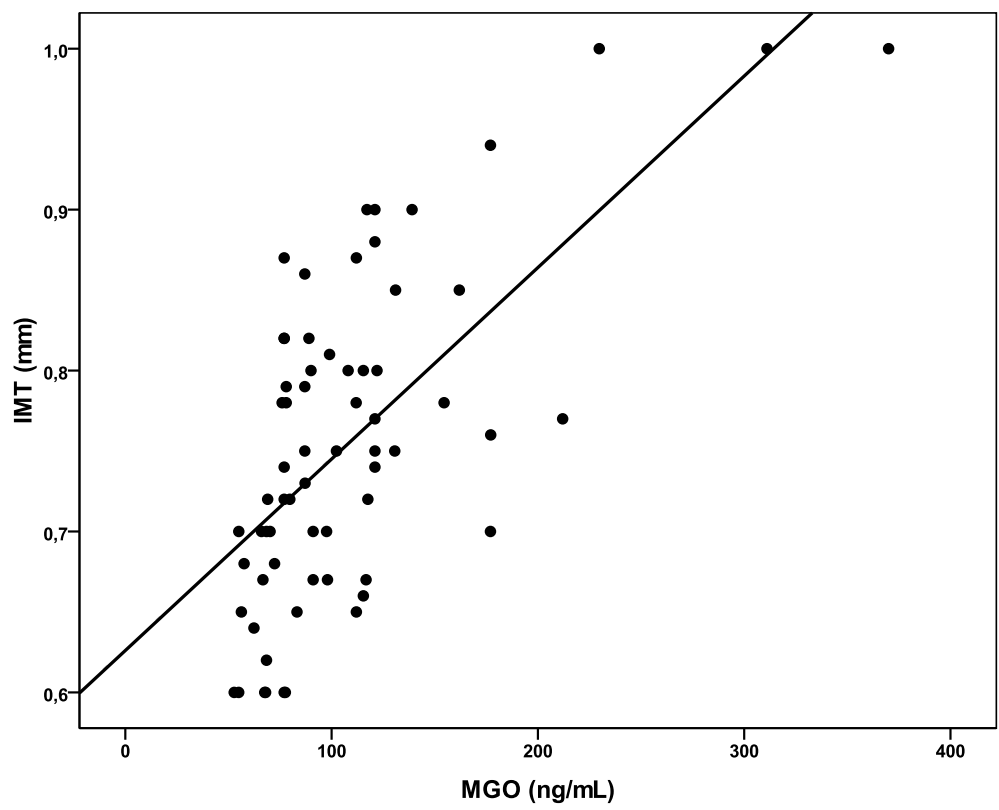

Figure 3. Scatter plot showing linear correlation between serum MGO and IMT in AR patients 


\section{Discussion}

The development of cardiovascular events is a major complication in patients with RA due to accelerated atherosclerosis. In RA, controlling systemic inflammation is currently viewed as an effective strategy for modifying CVD risk factors and preventing vascular events (Mason, 2015), because inflammation plays a major role in initiation and progression of atherosclerosis (Libby 2002). Carotid IMT is a non-invasive procedure as a useful marker of the progression of atherosclerosis and is associated with clinical CVD and has independent prognostic value and an excellent predictor of cardiovascular events (Roman, 2006, Roman, 2012). In a previous study carotid IMT demonstrated significant correlations with disease severity, CRP and ESR values in RA patients (Kumeda, 2002, Gonzalez-Gay 2005, Del Rincon, 2003).

Traditional risk factors such as smoking and dyslipidemia, which are best known for contributing to the development of atherosclerosis in early and active RA (Nurmohamed,2009), however, there are other non-traditional risk factors that recently are considered to be among critical pathogenic factors that appear to be involved in RA. Our previously studies have shown that serum CML and pentosidine levels were increased during RA and CML was independently associated with the presence and the severity of RA, and in other part, we found serum MGO and sRAGE (soluble receptor for advanced glycation end products) levels are inversely related to the activity of RA and increased serum MGO levels were independently associated with higher disease activity of RA (Knani, 2018a, 2018b). In addition to these new studies, we showed before that serum AGEs levels were increased in diabetes and its vascular complications, in particular the involvement of serum pentosidine levels in the development of atherosclerosis in coronary artery disease (Kerkeni, 2014). Yoshida et al, demonstrated that serum pentosidine concentrations showed a significant positive correlation with carotid IMT in patients with diabetes (Yoshida, 2005).

With respect to the serum AGEs measurement, there is a new noninvasive measurement of AGEs accumulation in the skin, named a skin autofluorescence (AF), has been recognized as a useful and convenient marker for diabetic vascular diseases (Meerwaldt 2004). Recently, some studies showed that skin AF as a surrogate marker for IMT in patients with diabetes (Temma,2015, Osawa,2017, Yashioka,2017) and the use of skin AF may help to accurately the real cardiovascular risk at the early stage of chronic kidney disease (Sanchez, 2017). Only, by skin AF measurement, one study has shown that in RA patients, the AGEs were correlated with IMT (de Groot, 2011). However, with our knowledge, there are no studies showing the relation between serum AGEs and IMT in RA patients. This is the first study that we showed serum pentosidine, CML and MGO levels are increased and are significantly related to IMT and serum CML and MGO were independently associated with IMT in RA patients.

Chronic inflammation process might result in the production of AGEs and AGEs precursors. AGEs can be a ligand for the receptor of AGE (RAGE), which is expressed on neutophils, macrophages, T-cells and synovial fibroblasts (Pullerits, 2005). The formed complex AGEs-RAGE activates the nuclear factor- $B$ that stimulates transcription of pro-inflammatory genes and leads to up-regulation of endothelial adhesion molecules and 


\section{Macrothink}

facilitates adhesion of circulating T-lymphocytes (Lander, 1997). As such, AGEs-RAGE interaction can become a mechanism process for developing atherosclerosis in RA (Carroll, 2006). We found that serum pentosidine, CML and MGO were positively related to IMT and serum CML and MGO were independently associated with IMT in RA, suggesting that not only AGEs are involved in the development of atherosclerosis plaques but also precursor of AGEs, MGO, is involved too. We speculate that in the RA patients increased serum AGEs and its precursors can be used as prognostic value for predicting IMT development.

\section{Conclusions}

This is a first study that shows an increased serum pentosidine, CML and MGO levels in RA patients. These glycated products were significantly related to IMT. This supports the hypothesis that in RA pathology, AGEs and its precursors may be early markers for cardiovascular disease and atherosclerosis development.

\section{Abbreviations}

AGEs: advanced glycation end products

Anti-CCP: anti-cyclic citrullinated peptides

BMI: body mass index

CML: carboxymethyllysine

CVD: cardiovascular disease

DAS28: disease activity score of 28 joins

ESR: erythrocyte sedimentation rate

EULAR: european league against rheumatism

HAQ: health assessment questionnaire

HDL: high density lipoprotein

hs-CRP: high-sensitive C-reactive protein

IgM-RF: imminoglobolin M-rheumatoid factor

IMT: intima media thickness

LDL: low density lipoprotein

MGO: methyglyoxal

RA: rheumatoid arthritis

\section{Declarations}

\section{Ethical approval and consent to participate}

Monastir Hospital public Fattouma Bourguiba ethics Committee (REF: Centre 0707) 
approved the study and informed consent was obtained from all study participants.

\section{Competing interests}

The authors declare that they have no competing interests

\section{Funding}

This work was supported by funds from Ministry of High Education and Scientific Research in Tunisia.

\section{Authors' contributions}

IK carried out the recruitment of RA patients and control subjects, the acquisition of the clinical data, the quantification of pentosidine, CML and MGO, the interpretation of data, and the statistical analysis and drafted the manuscript. HB carried out all biochemical laboratory measurement and technical assistance. AL participated in the preparation of the manuscript. JG participated in the design of the study. MK supervised the study and participated in the in the statistical analysis and in the writing of the manuscript.

\section{Acknowledgements}

We thank Monastir Hospital public Fattouma Bourguiba for giving authorization and clinical data about patients.

\section{References}

Agca, R., Heslinga, S. C., Rollefstad, S., et al (2017). EULAR recommendations for cardiovascular disease risk management in patients with rheumatoid arthritis and other forms of inflammatory joint disorders. Ann Rheum Dis., 76, 17-28.

https://doi.org/10.1136/annrheumdis-2016-209775

Aletaha, D., Neogi, T., Silman, A., Funovits, J., Felson, D. T., Bingham, C. O., Birnbaum, N. S. et al. (2010). Rheumatoid arthritis classification criteria, an American college of rheumatology/European league against rheumatism collaborative initiative. Ann Rheum Dis., 69, 1580-1588.

Ambrosino, P., Tasso, M., Lupoli, R., et al (2015). Non-invasive assessment of arterial stiffness in patients with rheumatoid arthritis: a systematic review and meta-analysis of literature studies. Ann Med., 47, 457-467. https://doi.org/10.3109/07853890.2015.1068950

Baghdadi, L. R., Woodman, R. J., Shanahan, E. M., et al (2015). The impact of traditional cardiovascular risk factors on cardiovascular outcomes in patients with rheumatoid arthritis: a systematic review and meta-analysis. PLoS One;

https://doi.org/10.1371/journal.pone.0117952

Carroll, L., Hannawi, S., Marwick, T., \& Thomas, R. (2006). Rheumatoid arthritis: links with cardiovascular disease and the receptor for advanced glycation end products. Wien Med Wochenschr, 156, 42-52. https://doi.org/10.1007/s10354-005-0242-9

de Groot, L., Hinkema, H., Westra, J., Smit, A. J., Kallenberg, C. G. et al (2011). Advanced 
glycation endproducts are increased in rheumatoid arthritis patients with controlled disease. Arthritis Research \& Therapy, 13, R205-214. https://doi.org/10.1186/ar3538

Del Rincon, I., Williams, K., Stern, M. P., et al (2003). Association between carotid atherosclerosis and markers of inflammation in rheumatoid arthritis patients and healthy subjects. Arthritis Rheum, 48, 1833-1840. https://doi.org/10.1002/art.11078

Fransen, J., \& van Riel, P. L. (2005). The disease activity score and the EULAR response criteria. Clin Exp Rheumatol, 39, 93-99.

Gasparyan, A. Y., Stavropoulos-Kalinoglou, A., Mikhailidis, D. P., et al (2010). The rationale for comparative studies of accelerated atherosclerosis in rheumatic diseases. Curr Vasc Pharmacol., 8, 437-49. https://doi.org/10.2174/157016110791330852

Gonzalez-Gay, M. A., Gonzalez-Juanatey, C., Pi neiro, A., et al (2005). High grade C-reactive protein elevation correlates with accelerated atherogenesis in patients with rheumatoid arthritis. J Rheumatol., 32, 1219-1223.

Gonzalez-Gay, M. A., Gonzalez-Juanatey, C., Vazquez-Rodriguez, T. R., et al (2008). Endothelial dysfunction, carotid intima-media thickness, and accelerated atherosclerosis in rheumatoid arthritis. Semin Arthritis Rheum., 38, 67-70.

https://doi.org/10.1016/j.semarthrit.2008.02.001

Haddad, M., Knani, I., Bouzidi, H., Berriche, O., Hammami, M., \& Kerkeni, M. (2016). Plasma Levels of Pentosidine, Carboxymethyl Lysine, Soluble Receptor for Advanced Glycation End Products, and Metabolic Syndrome: The Metformin Effect. Disease Markers, 8. https://doi.org/10.1155/2016/6248264

Im, C. H., Kim, N. R., Kang, J. W., et al (2015). Inflammatory burden interacts with conventional cardiovascular risk factors for carotid plaque formation in rheumatoid arthritis. Rheumatology;54:808-815. https://doi.org/10.1093/rheumatology/keu376

Kerkeni, M., Saidi, A., Bouzidi, H., Letaief, A., Ben, Y. S., \& Hammami, M. (2013). Pentosidine as a biomarker for microvascular complications in type 2 diabetic patients. Diabetes andVascular Disease Research 10, 239-245.

https://doi.org/10.1177/1479164112460253

Kerkeni, M., Saidi, A., Bouzidi, H., Letaief, A., Ben, Y. S., \& Hammami, M. (2012). Elevated serum levels of AGEs, sRAGE, and pentosidine in Tunisian patients with severity of diabetic retinopathy. Microvascular Research, 84, 378-383.

https://doi.org/10.1016/j.mvr.2012.07.006

Kerkeni, M., Weiss, I. S., Jaisson, S. et al (2014). Increased serum concentrations of pentosidine are related to presence and severity of coronary artery disease. Thrombosis Research;134: 633-638. https://doi.org/10.1016/j.thromres.2014.07.008

Knani, I., Bouzidi, H., Zrour, S., Bergaoui, N., Hammami, M., \& Kerkeni, M. (2018a). Increased serum concentrations of $\mathrm{N} \xi$-carboxymethyllysine are related to presence and 
severity of rheumatoid arthritis. Ann Clin Biochem, 55, 430-436.

https://doi.org/10.1177/0004563217733500

Knani, I., Bouzidi, H., Zrour, S., Bergaoui, N., Hammami, M., \& Kerkeni, M. (2018b). Methylglyoxal, a relevant marker of disease activity in patients with rheumatoid arthritis. Disease Markers, 6 pages. https://doi.org/10.1155/2018/8735926

Kramer, H. R., \& Giles, J. T. (2011). Cardiovascular disease risk in rheumatoid arthritis: progress, debate, and opportunity. Arthritis Care Res., 63, 484-499.

https://doi.org/10.1002/acr.20386

Kumar, A., Malaviya, A. N., Randhir, A., \& Singh, R. (2002). Validation of an Indian version of the Health Assessment Questionnaire in patients with rheumatoid arthritis. Rheumatology, 41, 1457-1459. https://doi.org/10.1093/rheumatology/41.12.1457

Kumeda, Y., Inaba, M., Goto, H., et al (2002). Increased thickness of the arterial intima-media detected by ultrasonography in patients with rheumatoid arthritis. Arthritis Rheum; 46, 1489-1497. https://doi.org/10.1002/art.10269

Lander, H. M., Tauras, J. M., Ogiste, J. S., Hori, O., Moss, R. A., Schmidt, A. M. (1997). Activation of receptor for advanced glycation end products triggers a p21(ras)-dependent mitogen-activated protein kinase pathway regulated by oxidant stress. J Biol Chem., 272, 17810-17814. https://doi.org/10.1074/jbc.272.28.17810

Libby, P., Ridker, P. M., \& Maseri, A. (2002). Inflammation and atherosclerosis. Circulation., 105, 1135-1143. https://doi.org/10.1161/hc0902.104353

Mason, J. C., \& Libby, P. (2015). Cardiovascular disease in patients with chronic inflammation: mechanisms underlying premature cardiovascular events in rheumatologic conditions. Eur Heart J., 36, 482-489c. https://doi.org/10.1093/eurheartj/ehu403

Mateen, S., Moin, S., Khan, A. Q., Zafar, A., \& Fatima, N. (2016). Increased Reactive Oxygen Species Formation and Oxidative Stress in Rheumatoid Arthritis. PloS one; https://doi.org/10.1371/journal.pone.0152925

Meerwaldt, R., Graaff, R., Oomen, P. H. N., Links, T P., Jager, J. J., Alderson, N. L., ... Smit, A. J. (2004). Simple non-invasive assessment of advanced glycation endproduct accumulation. Diabetologia 2004, 47, 1324-1330. https://doi.org/10.1007/s00125-004-1451-2

Nasiri, R., Field, M. J., Zahedi, M., \& Akbar, Moosavi-Movahedi, M. (2012). Comparative DFT Study To Determine if $\alpha$-Oxoaldehydes are Precursors for Pentosidine Formation. $J$. Phys. Chem., 116, 2986-2996. https://doi.org/10.1021/jp2104165

Nurmohamed, M. T. (2009). Cardiovascular risk in rheumatoid arthritis. Autoimmun Rev., 8, 663-667. https://doi.org/10.1016/j.autrev.2009.02.015

Osawa, S., Katakami, N., Kuroda, A., Takahara, M., Sakamoto, F. et al (2017). Skin autofluorescence is associated with early stage atherosclerosis in patients with type 1 diabetes. J Atheroscler Thromb., 24, 312-326. https://doi.org/10.5551/jat.35592 


\section{Al Macrothink}

Pullerits, R., Bokarewa, M., Dahlberg, L., \& Tarkowski, A. (2005). Decreased levels of soluble receptor for advanced glycation end products in patients with rheumatoid arthritis indicating deficient inflammatory control. Arthritis Res Ther., 7, R817-824.

https://doi.org/10.1186/ar1749

Riley, W. A., Barnes, R. W., Applegate, W. B. et al (1992). Reproducibility of noninvasive ultrasonic measurement of carotid atherosclerosis: the asymptomatic carotid artery plaque study. Stroke; 23, 1062-1068. https://doi.org/10.1161/01.STR.23.8.1062

Roman, M. J., Naqvi, T. Z., Gardin, J. M., Gerhard-Herman, M., Jaff, M., \& Mohler, E (2006). American Society of Echocardiography, Society of Vascular Medicine and Biology. Clinical application of noninvasive vascular ultrasound in cardiovascular risk stratification: a report from the American society of echocardiography and the society of vascular medicine and biology. J. Am Soc Echocardiogr, 19, 943-954. https://doi.org/10.1016/j.echo.2006.04.020

Roman, M. W., Polak, J. F., Kavousi, M., et al (2012). Carotid intima-media thickness progression to predict cardiovascular events in the general population (the PROG-IMT collaborative project): a meta-analysis of individual participant data. Lancet., 379, 2053-2062. https://doi.org/10.1016/S0140-6736(12)60441-3

Sanchez, E., Betriu, A., Arroyo, D., Lopez, C., Hernandez, M. et al (2017). Skin autofluorescence and subclinical atherosclerosis in mild to moderate chronic kidney disease: a case-control study. PloS ONE; https://doi.org/10.1371/journal.pone.0170778

Temma, J., Matsuhisa, M., Horie, T., Kuroda, A., Mori, H. et al (2015). Non-invasive measurement of skin autofluorescence as a beneficial surrogate marker for atherosclerosis in patients with type 2 diabetes. J Med Invest., 62, 126-129. https://doi.org/10.2152/jmi.62.126

Yashioka, K. (2017). Skin autoflurescence is a noninvasive surrogate marker for diabetic microvascular complications and carotid intima media thickness in Japanese patients with type 2 diabetes: a cross sectional study. Diabetes

Ther., https://doi.org/10.1007/s13300-017-0339-3

Yoshida, N., Okumura, K. I., \& Aso, Y. (2005). High serum pentosidine concentrations are associated with increased arterial stiffness and thickness in patients with type 2 diabetes. Metabolism Clinical and Experimental, 54, 345-350.

https://doi.org/10.1016/j.metabol.2004.09.014

\section{Copyright Disclaimer}

Copyright for this article is retained by the author(s), with first publication rights granted to the journal.

This is an open-access article distributed under the terms and conditions of the Creative Commons Attribution license (http://creativecommons.org/licenses/by/3.0/). 